\title{
A NOTE ON BUMP FUNCTIONS THAT LOCALLY DEPEND ON FINITELY MANY COORDINATES
}

\author{
M. FABIAN AND V. ZiZLER
}

\begin{abstract}
We show that if a continuous bump function on a Banach space $X$ locally depends on finitely many elements of a set $\mathrm{F}$ in $X^{*}$, then the norm closed linear span of $F$ equals to $X^{*}$. Some corollaries for Markuševič bases and Asplund spaces are derived.
\end{abstract}

Real valued functions with bounded nonempty support (bump functions in short) are important in analysis on Banach spaces. Bump functions that locally depend on finitely many coordinates play an important rôle in Banach space theory (see for example, [1]). The main result of this note is related to the Bishop-Phelps theorem on the density of norm attaining functionals.

Definition 1: Let $F$ be a set in $X^{*}$. A function $\varphi$ that maps a Banach space $X$ into $\mathbb{R} \cup+\infty$ and is such that the set $\{x \in X ; \varphi(x)<+\infty\}$ is open is said to locally depend on finitely many elements of $F$ if for every $x \in X$, where $\varphi(x)<+\infty$ there is a neighbourhood $U$ of $x$ in $X$, a finite collection $\left\{f_{1}, f_{2}, \ldots, f_{n}\right\}$ in $F$ and a continuous real valued function $\psi$ on $\mathbb{R}^{n}$ such that $\varphi(y)=\psi\left(f_{1}(y), \ldots, f_{n}(y)\right)$ for every $y \in U$. If $\left\{x_{\alpha}, f_{\alpha}\right\}_{\alpha \in \Gamma}$ is a Markuševič basis for a Banach space $X$ and a continuous bump function $\varphi$ locally depends on finitely many elements of $F=\left\{f_{\alpha}\right\}_{\alpha \in \Gamma}$, we say that a function $\varphi$ locally depends on finitely many coordinates of the Markuševič basis $\left\{x_{\alpha}, f_{\alpha}\right\}$. If $F=X^{*}$ and a bump function $\varphi$ on $X$ locally depends on finitely many elements of $F$, we say that $\varphi$ locally depends on finitely many functionals or coordinates.

Recall that a biorthogonal system $\left\{x_{\alpha}, f_{\alpha}\right\}_{\alpha \in \Gamma}$ is a Markuševič basis for a Banach space $X$ if the norm closed linear span of $\left\{x_{\alpha}\right\}_{\alpha \in \Gamma}$ equals $X$ and $\left\{f_{\alpha}\right\}_{\alpha \in \Gamma}$ separates the points of $X$ (that is, given $x \in X$, there is $\alpha \in \Gamma$ such that $f_{\alpha}(x) \neq 0$ ). If, moreover, the norm closed linear span of $\left\{f_{\alpha}\right\}_{\alpha \in \Gamma}$ equals to $X^{*}$, we speak of a shrinking Markuševič basis for $X$ (see for example, [2]).

Received 15th January, 1997.

The first author was supported by VČR 101-9502 and GAČR (Czech Republic). The second author was supported by 7926 NSERC (Canada).

Copyright Clearance Centre, Inc. Serial-fee code: 0004-9729/97 \$A2.00+0.00. 
It was shown in [5] (see for example, [1, Proposition V.2.5]) that an infinite dimensional Banach space $X$ contains a subspace isomorphic to $c_{0}$ if it admits a continuous bump function that locally depends on finitely many coordinates. Hájek showed in [3] that a separable Banach space $X$ admits an equivalent norm that away from the origin is $C^{\infty}$-smooth and locally depends on finitely many coordinates if $X$ admits an equivalent norm that away from the origin locally depends on finitely many coordinates. We do not know if Hájek's result can be extended for bump functions or for norms in the case of nonseparable $X$. It is known that a Banach space $X$ is an Asplund space (that is, each separable subspace of $X$ has separable dual) if it admits a Fréchet differentiable bump function (see for example, [1, Theorem II.5.3]). In this note we shall prove the result stated in Abstract, which implies that a Banach space is an Asplund space if it admits a continuous bump function that locally depends on finitely many coordinates. Also, we shall show that a Markuševič basis $\left\{x_{\alpha}, f_{\alpha}\right\}$ of a Banach space $X$ is necessarily shrinking if $X$ admits a continuous bump function that locally depends on finitely many coordinates of the Markuševič basis $\left\{x_{\alpha}, f_{\alpha}\right\}$. We shall prove that continuous bump functions depending locally on finitely many coordinates cannot exist on any infinite dimensional dual Banach space.

TheOREM 1. Let $X$ be a Banach space and let $F$ be a set in $X^{*}$. Assume that $\varphi$ is a continuous bump function on $X$ that locally depends on finitely many elements of $F$. Then the norm closed linear span of $F$ equals to $X^{*}$.

Proof: Define the function $\Phi$ on $X$ by

$$
\Phi(x)=\left\{\begin{array}{lll}
\varphi^{-2}(x) & \text { if } & \varphi(x) \neq 0 \\
+\infty & \text { if } & \varphi(x)=0
\end{array}\right.
$$

It follows that $\Phi$ is a bounded below lower semicontinuous function on $X$ such that $S:=\{x \in X ; \Phi(x)<+\infty\}$ is open and $\Phi$ locally depends on finitely many elements of $F$.

In order to show that the norm closed linear span of $F$ equals $X^{*}$, let $f \in X^{*}$ and $\varepsilon>0$ be given.

From the Ekeland variational principle (see for example, [1, Theorem I.2.4.]) it follows that there is $x_{0} \in S$ such that

$$
(\Phi-f)(x) \geqslant(\Phi-f)\left(x_{0}\right)-\varepsilon\left\|x-x_{0}\right\|
$$

for all $x \in X$.

Let $U, f_{1}, f_{2}, \ldots, f_{n} \in F$ and $\psi$ be as in Definition 1 for $\Phi-f$ and $x_{0}$.

Let

$$
W=\left\{x \in X ; f_{1}(x)=f_{2}(x)=f_{n}(x)=0\right\}
$$


Finally let $\delta>0$ be such that $x_{0}+h \in U$ whenever $h \in X$ is such that $\|h\|<\delta$.

Then if $h \in W,\|h\|<\delta$, we have

$$
\begin{aligned}
\Phi\left(x_{0}+h\right)-\Phi\left(x_{0}\right) & =\psi\left(f_{1}\left(x_{0}+h\right), \ldots, f_{n}\left(x_{0}+h\right)\right)-\psi\left(f_{1}\left(x_{0}\right), \ldots, f_{n}\left(x_{0}\right)\right) \\
& =\psi\left(f_{1}\left(x_{0}\right), \ldots, f_{n}\left(x_{0}\right)\right)-\psi\left(f_{1}\left(x_{0}\right), \ldots, f_{n}\left(x_{0}\right)\right) \\
& =0
\end{aligned}
$$

Hence from (1), for $h \in W,\|h\|<\delta$ we have

$$
f(h)=f\left(x_{0}+h\right)-f\left(x_{0}\right) \leqslant \Phi\left(x_{0}+h\right)-\Phi\left(x_{0}\right)+\varepsilon\|h\|=\varepsilon\|h\| .
$$

Let $\tilde{f}$ be the restriction of $f$ to $W$. Then by (2), for $\tilde{f}$ as an element of $W^{*}$, we have $\|\tilde{f}\| \leqslant \varepsilon$.

Let $\tilde{f}_{1}$ be a norm preserving Hahn-Banach extension of $\tilde{f}$ to $X$.

Note that $f-\tilde{f}_{1} \in W^{\perp}$ and it follows from the bipolar theorem that $W^{\perp}=$ $\operatorname{span}\left\{f_{1}, f_{2}, \ldots, f_{n}\right\}$ as finite dimensional subspaces in $X^{*}$ are weak star closed.

Hence

$$
\begin{aligned}
\operatorname{dist}(f, \operatorname{span} F) & \leqslant \operatorname{dist}\left(f, \operatorname{span}\left\{f_{1}, f_{2}, \ldots, f_{n}\right\}\right) \\
& =\operatorname{dist}\left(f, W^{\perp}\right) \\
& \leqslant \operatorname{dist}\left(f, f-\widetilde{f}_{1}\right) \\
& =\left\|\tilde{f}_{1}\right\| \\
& \leqslant \varepsilon
\end{aligned}
$$

Thus the norm closed linear span of $F$ equals $X^{*}$. Theorem 1 is proven.

Corollary 2. Assume that a Banach space $X$ admits a continuous bump function that locally depends on finitely many coordinates. Then $X$ is an Asplund space.

Proof: Let $\varphi$ with $\varphi(0) \neq 0$ be a continuous bump function on $X$ that locally depends on finitely many coordinates. Assume that $Y$ is a separable subspace of $X$. Let $\tilde{\varphi}$ be the restriction of $\varphi$ to $Y$. Then $\widetilde{\varphi}$ is a continuous bump function on $Y$ that locally depends on finitely many coordinates (use the restriction of functionals on $Y$ ).

By Definition 1, for every $x \in Y$ choose a neighbourhood $U_{x}$ in $Y$, a finite number of elements $\left\{f_{1}^{x}, f_{2}^{x}, \ldots, f_{n_{x}}^{x}\right\}$ of $Y^{*}$ and a continuous function $\psi^{x}$ on $\mathbb{R}^{n_{x}}$ such that $\Phi(z)=\psi^{x}\left(f_{1}^{x}(z), f_{2}^{x}(z), \ldots, f_{n_{x}}^{x}(z)\right)$ for every $z \in U_{x}$. By the Lindelöf property, let $\left\{x_{n}\right\}_{n=1}^{\infty} \subset Y$ be such that $\left\{U_{x_{n}}\right\}_{n=1}^{\infty}$ covers $Y$.

For $n \in \mathbb{N}$ let $F_{n}=\left\{f_{1}^{x_{n}}, f_{2}^{x_{n}}, \ldots, f_{n_{x_{n}}}^{x_{n}}\right\}$. Denote by $F=\bigcup_{n=1}^{\infty} F_{n}$. 
Then $\widetilde{\varphi}$ is a continuous bump function on $Y$ that locally depends on finitely many elements of $F$ and $F$ is countable. By Theorem $1, Y^{*}$ is a norm closed linear span of $F$ and thus $Y^{*}$ is norm separable. Hence $X$ is an Asplund space. Corollary 2 is proven.

Corollary 3. Assume that $\left\{x_{\alpha}, f_{\alpha}\right\}_{\alpha \in \Gamma}$ is a Markuševič basis for a Banach space $X$. Assume that $X$ admits a continuous bump function that locally depends on

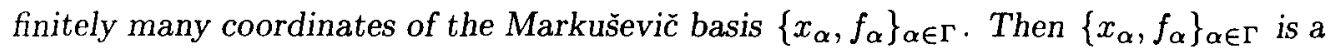
shrinking Markuševič basis for $X$.

Proof: It suffices to use Theorem 1 for $F=\left\{f_{\alpha}\right\}_{\alpha \in \Gamma}$.

Corollary 4. Assume that an infinite dimensional Banach space $X$ admits a continuous bump function that locally depends on finitely many coordinates. Then $X$ is not isomorphic to a dual Banach space.

ProOF: Assume that $X$ is isomorphic to $Y^{*}$ for some Banach space $Y$. By the result in [5] (see [1, Proposition V.2.5]), $X$ contains a subspace isomorphic to $c_{0}$. Thus $Y^{*}$ contains a subspace isomorphic to $\ell_{\infty}$ by the result of Bessaga and Pelczynski (see for example, [4, Proposition 2.e.8]). The space $X$ is an Asplund space by Corollary 2. Hence $Y^{*}$ is an Asplund space and thus $\ell_{\infty}$ is an Asplund space. This is not true as $\ell_{\infty}$ contains a subspace isometric to $\ell_{1}$ the dual of which is nonseparable. Corollary 4 is proven.

Corollary 3 can be compared with the fact that a monotone Schauder basis $\left\{x_{n}\right\}$ for a Banach space $X$ with the Fréchet differentiable norm is necessarily shrinking (see for example, [2, Proposition 240]). The canonical supremum norm of $c_{0}$ is a typical example of a norm that away from the origin locally depends on finitely many coordinates of the standard Schauder basis for $c_{0}$. If $\left\{x_{n}, f_{n}\right\}$ is a nonshrinking Schauder basis for $c_{0}$ (see for example, $\left[4\right.$, p.10]), then it follows from Corollary 3 that $c_{0}$ admits no continuous bump function that locally depends on finitely many coordinates of the basis $\left\{x_{n}, f_{n}\right\}$.

\section{REFERENCES}

[1] R. Deville, G. Godefroy and V. Zizler, Smoothness and renormings in Banach spaces, Pitman Monographs and Surveys in Pure and Applied Mathematics 64 (Longman, Harlow, 1993).

[2] P. Habala, P. Hájek and V. Zizler, Introduction to Banach spaces I, II, (Lecture Notes) (Matfyzpress, Charles University, Prague, 1996).

[3] P. Hájek, 'Smooth norms that depend locally on finitely many coordinates', Proc. Amer. Math. Soc. 123 (1995), 3817-3821.

[4] J. Lindenstrauss and L. Tzafriri, Classical Banach spaces I, Sequence spaces (SpringerVerlag, Berlin, Heidelberg, New York, 1977). 
[5] J. Pechanec, J.H.M. Whitfield and V. Zizler, 'Norms locally dependent on finitely many coordinates', An. Acad. Brasil. Ciênc. 53 (1981), 415-417.

Mathematical Institute

Czech Academy of Sciences

Žitná 25, 11567 Prague 1

Czech Republic

e-mail: fabian@karlin.mff.cuni.cz
Department of Mathematical Analysis Faculty of Mathematics and Physics Charles University

Sokolovská 83, 18600 Prague 8

Czech Republic

and

Department of Mathematics

University of Alberta, Edmonton

T6G 2G1 Alberta

Canada

e-mail: vzizler@vega.math.ualberta.ca 